Research Paper

\title{
Photophysics and Rotational Dynamics of a Fluoroquinolone Drug Norfloxacin in Biomimetic Reverse Micellar Nanocavities
}

\author{
BIJAN KUMAR PAUL, NARAYANI GHOSH, ANU TEWARY and SAPTARSHI MUKHERJEE* \\ Department of Chemistry, Indian Institute of Science Education and Research Bhopal, Bhopal Bypass \\ Road, Bhauri, Bhopal 426 066, Madhya Pradesh, India
}

(Received on 23 November 2015; Revised on 04 March 2016; Accepted on 16 March 2016)

\begin{abstract}
The present work describes the study of the photophysics and dynamics of a prospective anti-cancer antibacterial drug norfloxacin (NFX) within the micro-heterogeneous environment of Aerosol OT (AOT) reverse micelle (RM). The environment sensitive fluorescence spectroscopic behavior of NFX is found to exhibit a significant red-shift with increasing water pool $\operatorname{size}\left(\mathrm{w}_{0}=\right.$ [water $] /[$ surfactant $\left.]\right)$ of the RM. A prominent decrease of fluorescence anisotropy of NFX with increasing $\mathrm{w}_{0}$ demonstrates a relaxation of the motional restrictions imposed on the bound drug molecules with enhanced hydration. These results are further substantiated from our time-resolved measurements. Study of the rotational dynamics of NFX within the $\mathrm{RM}$ as a function of $\mathrm{w}_{0}$ exhibits a typical biexponential decay behavior which has been adequately described on the basis of the wobbling-in-cone model. The rotational dynamical parameters are also calculated for wobbling motion of the drug within the RM as a function of $\mathrm{w}_{0}$.
\end{abstract}

Keywords: Norfloxacin; Antibacterial Drug; AOT Reverse Micelle; Fluorescence Spectroscopy; Rotational Dynamics; Wobbling-in-Cone

\section{Introduction}

Reverse micelles (RMs; Scheme 1) are spherical aggregates formed by surfactant molecules in nonpolar medium and consist of a solvent water pool entrapped in the core of the reverse micellar unit surrounded by the headgroups of surfactants with the non-polar hydrocarbon chains of surfactants projected toward the non-polar bulk medium (Levinger et al., 2002; Bhattacharyya et al., 2003; Baruah et al., 2006; Willard et al., 1998; Silber et al., 1999; Satpati et al., 2009). The water molecules entrapped inside the reverse micelle are characterized to be highly structured and non-homogeneous in nature. The average size of a reverse micelle is dependent on the amount of solubilized water and expressed by water to surfactant molar ratio, $\mathrm{w}_{0}=[$ water $] /[$ surfactant $]$ (Luisi et al., 1984; Kalyansundaram et al., 1987; Luisi et al., 1988; Jain et al., 1989). Several studies have shown that two types of water population exist inside the water pool (Levinger et al., 2002; Bhattacharyya et al., 2003; Baruah et al., 2006; Willard et al., 1998), namely, water molecules that are strongly bound with the headgroup of the surfactant and hence display distinct characteristics compared to bulk water (Dutt et al., 2004) and the free water molecules that reside deep inside the core of the water pool. Thus the interior of a reverse micelle is truly heterogeneous. Reverse micelles are widely considered as an efficient model for biological systems because of their ability to mimic several essential and important features of biological membranes. Given the structural, compositional and functional complexities associated with biological membranes, researchers have been particularly motivated to find some simple model system to mimic the physicochemical properties of membranes (Fendler et al., 1976; Mitra et al., 2008; Faeder et al., 1989; Venables et al., 2001; Nandi et al., 2000; Bhattacharyya et al., 2000). In our case we have used aerosol OT (AOT) to prepare the reverse micellar system. AOT is a double tailed anionic surfactant which is used for solubilization and

*Authorfor Correspondence: E-mail: saptarshi@iiserb.ac.in 
emulsification. It can form spherical aggregates in wide range of $\mathrm{w}_{0}$ values (Nave et al., 2000), that is, the AOT reverse micelle can compartmentalize large amount of water into its core.

Fluoroquinolone (FQ) classes of compounds are widely known for their broad spectrum antibacterial activities. FQs are used as antibacterial drugs in the treatment of various infectious diseases (Lipsky et al., 1999; Stahlmann et al., 1999; Anand et al., 2012), sexually transmitted diseases such as prostatitis, skin infection, acute cystitis and selected pneumonias (Ting et al., 2003; Appelbaum et al., 2000; Ball et al., 2000; Blondeau et al., 1999). The mechanism of action of FQs with biological or biomimicking assemblies has been quite unique in the sense that both the hydrophilic as well as hydrophobic pathways have been invoked as the actual mechanistic conduits, and the latter being mainly responsible for the route leading to their entry into the cytoplasm (Nikaido et al., 1993; Berlanga et al., 2000).
Herein, we have carried out a spectroscopic investigation of interaction of a fluoroquinolone drug, norfloxacin (NFX; Scheme 1) with AOT reverse micellar system. The chemical structure of NFX is comprised of a carbonyl group and a piperazinyl moiety at the 7-carbon atom. The 4' ' $\mathrm{N}$ ' of the piperazinyl ring and the carboxylate group are the most significant proton binding sites from the biological viewpoint which leads to $\mathrm{pH}$-dependent prototropism which in NFX is characterized by two $\mathrm{pK}_{\mathrm{a}}$ values, $\mathrm{pK}_{1}=6.23$ and $\mathrm{pK}_{2}=8.55$ (Albini et al., 2003; Sortino et al., 1998; Monti et al., 2002; Paul et al., 2015).

In this study, we have studied the photophysical properties and rotational dynamics of NFX in AOT reverse micelle with various $\mathrm{w}_{0}$ values by using steady-state and time-resolved fluorescence spectroscopic techniques. The photophysical and spectroscopic data are very useful in elucidating the interaction of these drug molecules with relevant biological and biomimicking assemblies. Upon<smiles>CCN1CC(C(=O)O)C(=O)c2cc(F)c(N3CCNCC3)cc2N1CC</smiles>

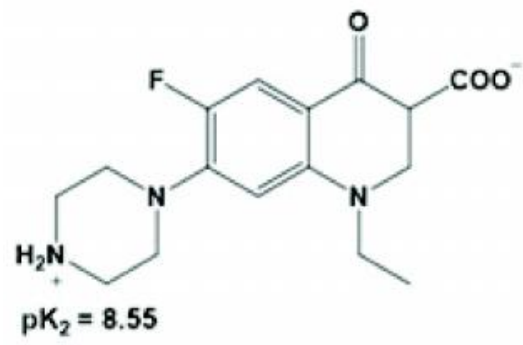

Zwitterion

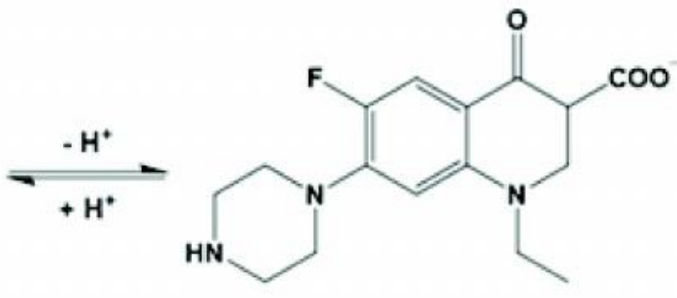

Anion

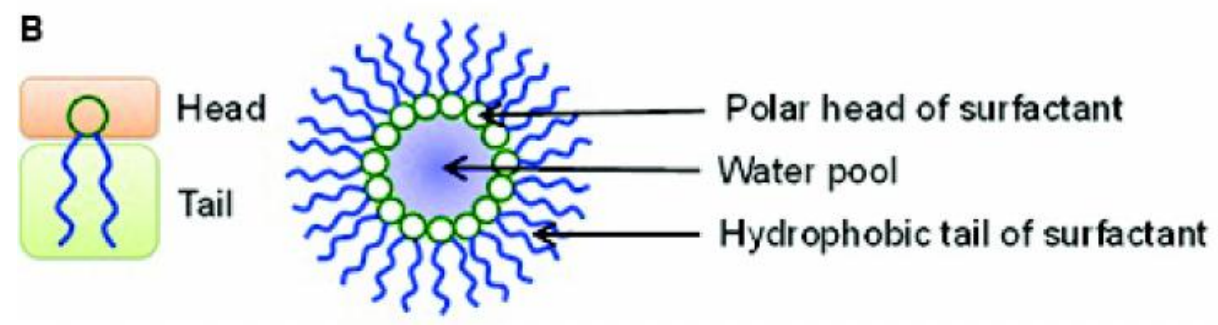

Scheme 1: (A) Prototropic Equilibrium of NFX ( $\mathrm{pK}_{1}=6.23$ and $\left.\mathrm{pK}_{2}=8.55\right)$ and (B) Simplified Cartoon Representation of a Reverse Micellar System 
entrapment inside an organized molecular assembly, the drug molecules will experience restriction on its dynamics and mobility. Fluorescence depolarization measurement yields an estimation regarding the dynamical information about the fluorophore in the complex micro-heterogeneous environment such as the reverse micelle. Understanding the rotational behavior of a probe in the microheterogeneous medium is significant for determining the dynamics of drug in biological systems. Hence, in the present study we intend to explore the dynamics of the drug (NFX) within the anionic RMs to understand their rotational behavior in such organized assemblies.

\section{Materials and Methods}

Norfloxacin (NFX) (Scheme 1) and sodium bis (2ethylhexyl) sulfosuccinate (AOT) from SigmaAldrich, USA and spectroscopic grade n-heptane from Spectrochem, India were used as procured. Triply distilled deionized Milli pore water was used for all measurements.

\section{Steady-State Spectral Measurements}

The absorption and fluorescence emission (and excitation) spectra were acquired on a Cary $100 \mathrm{UV}$ Vis spectrophotometer and Fluorolog 3-111 fluorometer, respectively. In all measurements the concentration of NFX was maintained at $c a .2 .0 \mathrm{mM}$ in order to avoid aggregation and reabsorption effects. Only freshly prepared solutions were used for spectroscopic measurements. Appropriate spectral background correction and instrumental correction have been ensured during all spectral measurements.

\section{Steady-State Fluorescence Anisotropy}

Steady-state anisotropy (r) was recorded on Fluorolog 3-111 fluorometer. The steady-state anisotropy (r) is defined as (Lakowicz, 1999):

$$
\begin{aligned}
& r=\frac{I_{V V}-G \cdot I_{V H}}{I_{V V}+2 . G I_{V H}} \\
& G=\frac{I_{H V}}{I_{H H}}
\end{aligned}
$$

where, $I_{\mathrm{VV}}$ and $I_{\mathrm{VH}}$ are the emission intensities when the excitation polarizer is vertically oriented and the emission polarizer is oriented vertically and horizontally, respectively. $\mathrm{G}$ is the correction factor.

\section{Time-Resolved Measurements}

Fluorescence lifetimes were obtained by the method of Time Correlated Single Photon Counting (TCSPC) following excitation of the samples at $\lambda_{\mathrm{ex}}=340 \mathrm{~nm}$ (IBH-NanoLED-340, FWHM 750 ps) using picosecond diodes and the signals were collected using a Hamamatsu MCP Photomultiplier (Model R-3809U50 ) at the magic angle polarization of $54.7^{\circ}$ to eliminate any considerable contribution from fluorescence anisotropy decay (Lakowicz, 1999). The decays were deconvoluted on DAS-6 decay analysis software. The average lifetime $\langle\tau\rangle$ is calculated using the following equation (Lakowicz, 1999):

$$
\langle\tau\rangle=\sum_{i} \alpha_{i} \tau_{i}
$$

where, $\alpha_{i}$ is the normalized pre-exponential factor for the $\mathrm{i}^{\text {th }}$ lifetime component.

For time-resolved fluorescence anisotropy decay measurements, the polarized fluorescence decays for the parallel $\left[I_{\|}(t)\right]$ and the perpendicular $\left[I_{\perp}(t)\right]$ emission polarizations with respect to the vertical excitation polarization were first collected at the emission maxima of the samples under investigation. The anisotropy decay function, $r(t)$ was then constructed using these $I_{\|}(t)$ and $I_{\perp}(t)$ decays on the following equation (Lakowicz, 1999):

$$
r(t)=\frac{I_{\|}(t)-G I_{\perp}(t)}{I_{\|}(t)+2 G I_{\perp}(t)}
$$

where, $\mathrm{G}$, the grating factor was determined from long time tail matching technique (Lakowicz, 1999).

\section{Results and Discussion}

\section{Steady-State Fluorescence Study}

Before discussing the spectral changes of NFX inside AOT reverse micelles, we first focus on the photophysical properties of the drug in bulk solvents which could serve as a comparative avenue for assessing the changes in spectral properties of the drug within the AOT reverse micellar assembly. NFX exhibits highly environment-sensitive spectral properties because of the $\mathrm{pH}$-dependent protropic equilibrium (Scheme 1). 
Figure 1a exhibits the absorption spectra of NFX in aqueous solution of different $\mathrm{pH}(\mathrm{s})$. The absorption profile of $\mathrm{NFX}$ at $\mathrm{pH}=7.4$ shows a strong absorption at $\sim 270 \mathrm{~nm}$ due to the presence of the aromatic ring and another absorption band in the $300-350 \mathrm{~nm}$ wavelength region with two sub-peaks at $\sim 325 \mathrm{~nm}$ and $335 \mathrm{~nm}$. This long wavelength absorption is ascribed to the $\mathrm{n} \rightarrow \pi^{*}$ electronic transition (Albini $e t$ al., 2003; Paul et al., 2015). With decrease in $\mathrm{pH}$ of the solution from $\mathrm{pH}=7.4$ to an acidic value $(\mathrm{pH}=$ 4.0), the short wavelength absorption band is found to undergo a red-shift by $\sim 5 \mathrm{~nm}$ (from $\sim 270 \mathrm{~nm}$ to $\sim$ $275 \mathrm{~nm}$ ), along with the appearance of a tail at the long wavelength region (beyond $350 \mathrm{~nm}$ ) (Fig. 1A). The absorption spectral changes to this direction have been identified with the formation of the cationic species of NFX in acidic pH (Albini et al., 2003). Figure 1a also shows a considerable decrease of absorption of NFX at alkaline $\mathrm{pH}(\mathrm{pH}=10.7)$, in analogy to literature reports (Albini et al., 2003; Paul et al., 2015).

Figure $1 \mathrm{~b}$ represents the emission spectra of NFX in aqueous medium of different $\mathrm{pH}(\mathrm{s})$. The emission profile of NFX at $\mathrm{pH}=4.0$ (i.e., acidic $\mathrm{pH}$ ) shows maxima at $\lambda_{\mathrm{em}} \sim 445 \mathrm{~nm}$ which is characteristic emission of the cationic species. The emission wavelength at $\lambda_{\mathrm{em}} \sim 418 \mathrm{~nm}$ in $\mathrm{pH} 7.4$ (i.e., neutral $\mathrm{pH})$ provides the signature of the zwitterionic form of NFX (Paul et al., 2015). At $\mathrm{pH}=10.7$ the emission spectra of NFX is centered at $\sim 420 \mathrm{~nm}$ (Fig. 1B) and the fluorescence yield of NFX at alkaline $\mathrm{pH}(\mathrm{pH}$ $=10.7$ ) is significantly low. Fluoroquinolone derivatives, e.g., NFX, are also known to exhibit solvent-dependent charge transfer emission from the nitrogen center of the piperazinyl group to the keto oxygen following photo-excitation (Blondeau et al., 1999; Nikaido et al., 1993; Berlanga et al., 2000; Albini et al., 2003).

Figure 1C represents the emission spectra of NFX in various solvents of different polarity. In chloroform $\left(\mathrm{CHCl}_{3}\right)$ and 1, 4-dioxane, NOF shows emission maxima at $\sim 404 \mathrm{~nm}$ attributable to the neutral species (Albini et al., 2003). In aqueous medium, NFX exhibits characteristic emission from the cationic species having emission maxima at $\sim 442 \mathrm{~nm}$. Thus, the maximum emission wavelength of NFX at $\sim 434$ $\mathrm{nm}$ in methanol is close to that of the cationic species of NFX.
The fluorescence profile of NFX in AOT reverse micelle with increasing water pool size (designated by $\mathrm{w}_{0}$ value) is displayed in Fig. 2. A progressive redshift of the emission wavelength with increasing $\mathrm{w}_{0}$ value indicates increase of polarity in the vicinity of the drug microenvironment. In this context, it is intriguing to note that the emission wavelength of NFX undergoes a remarkable red-shift to $\lambda_{\mathrm{em}} \sim 442 \mathrm{~nm}$ at $\mathrm{w}_{0}=22$ from $\lambda_{\mathrm{em}} \sim 408 \mathrm{~nm}$ at $\mathrm{w}_{0}=1$. On the basis of a direct comparison of the spectral properties of NFX in bulk solvents it can be argued that at low $\mathrm{w}_{0}$ the probe experiences a predominantly hydrophobic environment in which the neutral form of NFX is preferentially stabilized (as indicated by the characteristic fluorescence maxima, $\lambda_{\mathrm{em}} \sim 408 \mathrm{~nm}$ at $\mathrm{w}_{0}=1$, which is close to that of the neutral species of $\mathrm{NFX}, \lambda_{\mathrm{em}} \sim 404 \mathrm{~nm}$ in $\mathrm{CHCl}_{3}$ (Paul et al., 2015; Blondeau et al., 1999; Nikaido et al., 1993; Berlanga et al., 2000; Albini et al., 2003). Whereas, with increased hydration (high $\mathrm{w}_{0}$ ) the microenvironment surrounding the probe becomes considerably hydrophilic in which the cationic form of NFX appears to be preferentially stabilized (as designated by the characteristic fluorescence maxima, $\lambda_{\mathrm{em}} \sim 442 \mathrm{~nm}$ at $\mathrm{w}_{0}=22$, which closely resemble the characteristic cationic emission of NFX, $\lambda_{\mathrm{em}} \sim 445 \mathrm{~nm}$ in acidic $\mathrm{pH}$ (Fig. 1) (Paul et al., 2015; Blondeau et al., 1999; Nikaido et al., 1993; Berlanga et al., 2000; Albini et $a l ., 2003)$. However, it is important to note in this context that besides polarity of the micro-environment, change of $\mathrm{pH}$ in the locality of the drug within the AOT reverse micelle could also play a role in modulating the photophysics of the bound drug. A critical discussion on the matter has been attempted based on some specifically designed experimental results in a forthcoming section. Photophysics of the drug Using the anthroloxy probe of varying chain length, Kelkar et al. (Kelkar et al., 2004) have shown that the shift of the emission maxima in reverse micellar micro-heterogeneous assemblies is largely governed by its precise location in the RMs. They have reported that the emission maxima of 7-nitorbenz2-oxa-1,3-diazol-4-yl (NBD)-cholesterol is independent of $\mathrm{w}_{0}$ indicating that the probe is localized away from the core of the RM which is characterized by reduced water penetration (Kelkar et al., 2004). In parity with earlier studies (Kelkar et al., 2004; Sengupta et al., 2013), the possibility of localization of the probe in the bulk solvent (n-heptane) can be 

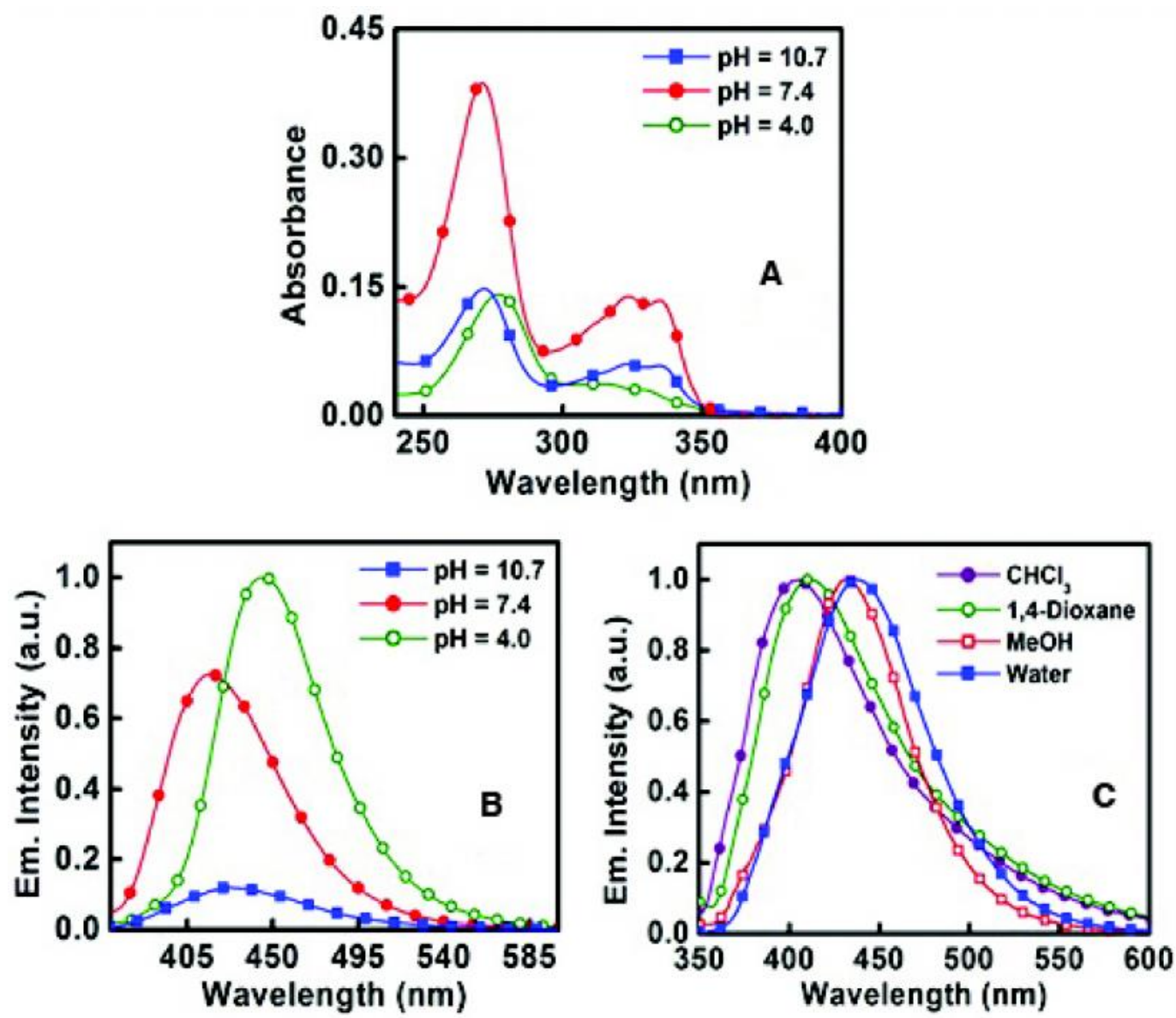

Fig. 1: (A) Absorption spectra of NFX in bulk aqueous solvent of varying $\mathrm{pH}(\mathrm{s})$ as indicated in the figure legend (pH = 4.0: $-\mathrm{o}-$ , pH = 7.4: -๑-, pH = 10.7: - - -), (B) Fluorescence spectra of NFX in bulk aqueous solvent of varying pH(s) as indicated in the figure legend $(\mathrm{pH}=4.0:-0-, \mathrm{pH}=7.4:-\bullet-, \mathrm{pH}=10.7:-\square-)$. $\lambda_{\mathrm{ex}}=335 \mathrm{~nm},[\mathrm{NFX}]=2.0 \mu \mathrm{M}$ and (C) Fluorescence

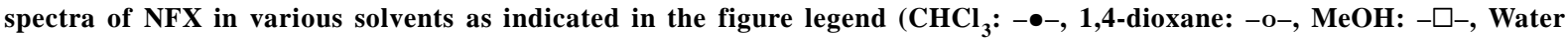
$(\mathbf{p H}=\mathbf{6 . 0})$ : - $\mathbf{-}-)$.

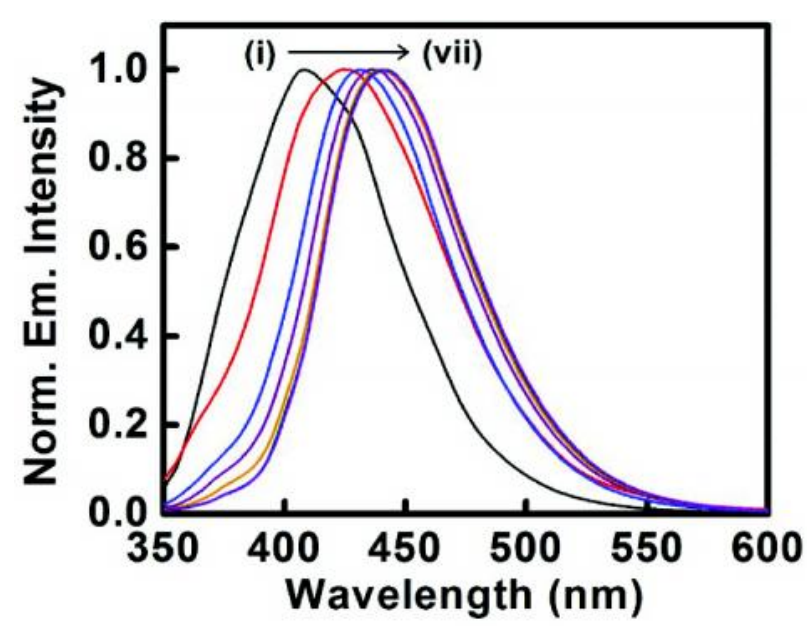

Fig. 2: Normalized fluorescence spectra of NFX $\left(\lambda_{e x}=335\right.$ $\mathrm{nm},[\mathrm{NFX}]=2.0 \mu \mathrm{M})$ in $\mathrm{AOT} / \mathrm{n}$-heptane reverse micelles as a function of $w_{0}$. Curves (i) () (vii) represent $\mathrm{w}_{0}=1,2,4,6,10,20,22$ ruled out with a view to the observed marked shift in the emission wavelength as a function of the water pool size $\left(\mathrm{w}_{0}\right.$ value). It is also noted that the extent of red-shift of NFX gradually decreases with increasing $\mathrm{w}_{0}$ and the variation of fluorescence wavelength almost attains saturation beyond $\mathrm{w}_{0}=15$ (Fig. 2). This can be rationalized based on the fact that the compartmentalized water in the water pool develops bulk type properties with increase of $\mathrm{w}_{0}$ value. Calorimetric studies (Goto et al., 1992), determination of dielectric constant (Biswas et al., 2008) and micropolarity studies inside the reverse micellar water pool (Biswas et al., 2008) have demonstrated that beyond $\mathrm{w}_{0} \sim 10$, the bulk-like properties become prominent in the water pool.

\section{Fluorescence Anisotropy Study}

The photophysical properties of a given drug molecule 
in a micro-heterogeneous assembly is largely governed by its precise location. The fluorescence anisotropy can function as sensitive indicator for any modification in rigidity of the surrounding environment of the fluorophore (Paul et al., 2015; Paul et al., 2014). In the present study, the fluorescence anisotropy of NFX is found to be higher inside the reverse micellar microenvironments at all $\mathrm{w}_{0}$ values compared to that in bulk water ( $\mathrm{r} \sim 0.013$ ) (Paul et al., 2015). This result suggests the impartation of motional restriction on the drug molecules inside the AOT RM (Fig. 3).

It is noted that the fluorescence anisotropy of the drug exhibits a specific variation as a function of $\mathrm{w}_{0}$ value in RM-encapsulated state (Fig. 3). With increase of $\mathrm{w}_{0}$ value, the anisotropy regularly decreases followed by a gradual saturation. This suggests the impartation of gradual relaxation on motional degrees of freedom of the probe with increasing $\mathrm{w}_{0}$ inside the RM. This observation probably indicates that the drug molecules move closer to the water pool with increasing $\mathrm{w}_{0}$ value. These findings are found to be in parity with the results reported (Sengupta et al., 2000).

\section{Time-Resolved Fluorescence Decay}

The typical time-resolved fluorescence decay profiles of NFX in RMs as a function of $\mathrm{w}_{0}$ value are displayed in Fig. 4 and the relevant decay parameters are collected in Table 1. NFX is found to exhibit bi-

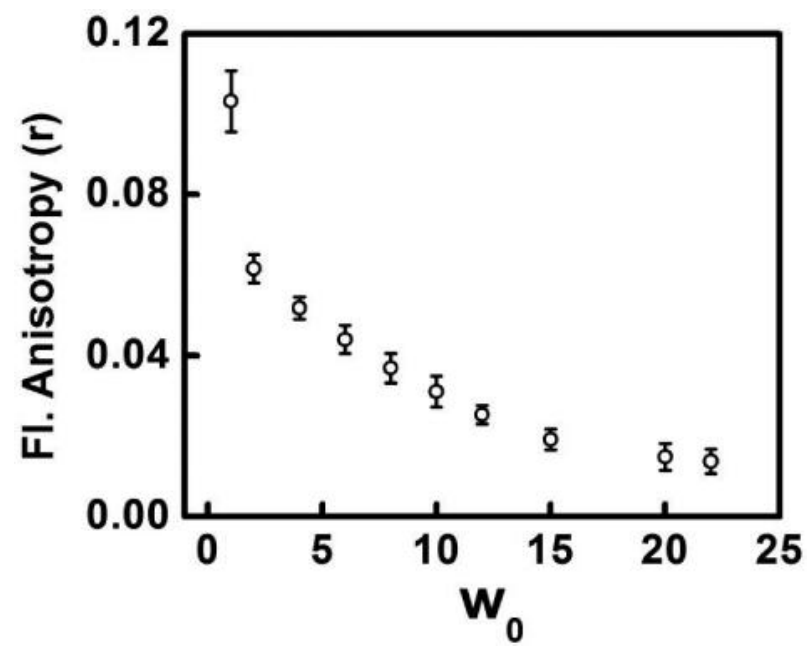

Fig. 3: Variation of steady-state fluorescence anisotropy of $\operatorname{NFX}\left(\lambda_{\text {ex }}=335 \mathrm{~nm},[\mathrm{NFX}]=\mathbf{2 . 0} \mu \mathrm{M}\right)$ in AOT/n-heptane reverse micelles as a function of $w_{0}$. Each data point is an average of 15 individual measurements exponential decay pattern within the AOT RMs (Table 1), however, the mean (average) fluorescence lifetime is used to explore the behavior of the drug in the microheterogeneous reverse micellar environments in various water pool sizes (it is indeed a non-trivial task to assign specific mechanistic models to each decay component in a heterogeneous environment). A gradual decrease of the average fluorescence lifetime of NFX with increasing $\mathrm{w}_{0}$ (Table 1) points out the fact that with increasing $\mathrm{w}_{0}$ swelling of the RMs becomes increasingly prominent which in turn accompanies decrease of motional constraint on the bound drug molecules. These results are found to be in keeping with the steady-state results as discussed above. The data summarized in Table 1 show that the

Table 1: Time-resolved fluorescence decay parameters of NFX in AOT reverse micelle with icreasing $w_{0}$

\begin{tabular}{lcccccc}
\hline $\mathrm{w}_{0}$ & $\tau_{1}^{\mathrm{a}}(\mathrm{ns})$ & $\tau_{2}{ }^{\mathrm{a}}(\mathrm{ns})$ & $\alpha_{1}$ & $\alpha_{2}$ & $\langle\tau\rangle(\mathrm{ns})$ & $\chi^{2}$ \\
\hline 1 & 1.31 & 4.81 & 0.35 & 0.65 & 3.59 & 1.07 \\
2 & 1.22 & 4.38 & 0.30 & 0.70 & 3.43 & 1.08 \\
4 & 1.10 & 4.16 & 0.24 & 0.76 & 3.44 & 1.02 \\
6 & 1.02 & 3.89 & 0.22 & 0.78 & 3.25 & 1.05 \\
8 & 0.99 & 3.64 & 0.17 & 0.83 & 3.18 & 1.06 \\
10 & 1.02 & 3.50 & 0.35 & 0.65 & 2.64 & 1.01 \\
12 & 1.04 & 3.38 & 0.17 & 0.83 & 2.99 & 1.09 \\
15 & 1.01 & 3.27 & 0.22 & 0.78 & 2.77 & 1.00 \\
22 & 1.00 & 3.19 & 0.34 & 0.66 & 2.45 & 1.01 \\
\hline${ }_{ \pm} 4 \%$ & & & & & &
\end{tabular}

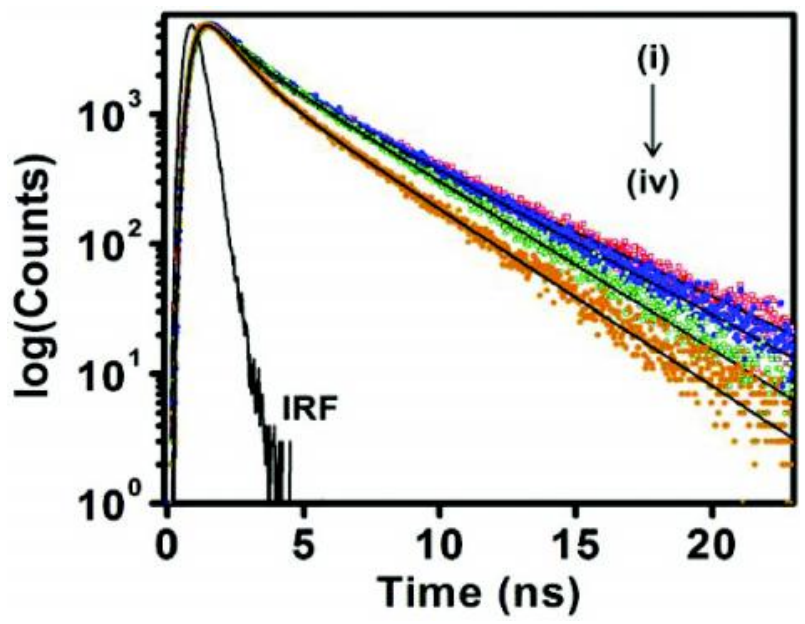

Fig. 4: Time-resolved fluorescence decay transients of NFX $\left(\lambda_{e x}=340 \mathrm{~nm},[\mathrm{NFX}]=2.0 \mu \mathrm{M}\right)$ in AOT/n-heptane reverse micelle with varying $w_{0}$. Curves (i) $\rightarrow$ (iv) indicate $w_{0}=2,6,12,22$ 
average lifetime of the probe even at the highest $\mathrm{w}_{0}$ value studied $\left(\tau=2.45 \mathrm{~ns}\right.$ at $\left.\mathrm{w}_{0}=22\right)$ is greater than that in aqueous medium $(\tau=1.91 \mathrm{~ns})$ and thus suggesting a more restricted environment of NFX within the RMs compared to bulk aqueous phase.

\section{Time-Resolved Fluorescence Anisotropy Decay}

To obtain further information about rotational relaxation of the fluorophore and the microenvironment around the fluorophore in reverse micellar environment time-resolved fluorescence anisotropy measurement has been performed as a function of water pool size in RM. The typical anisotropy decay profiles of NFX in RMs at various $\mathrm{w}_{0}$ values are presented in Fig. 5 and the relevant parameters are summarized in Table 2.
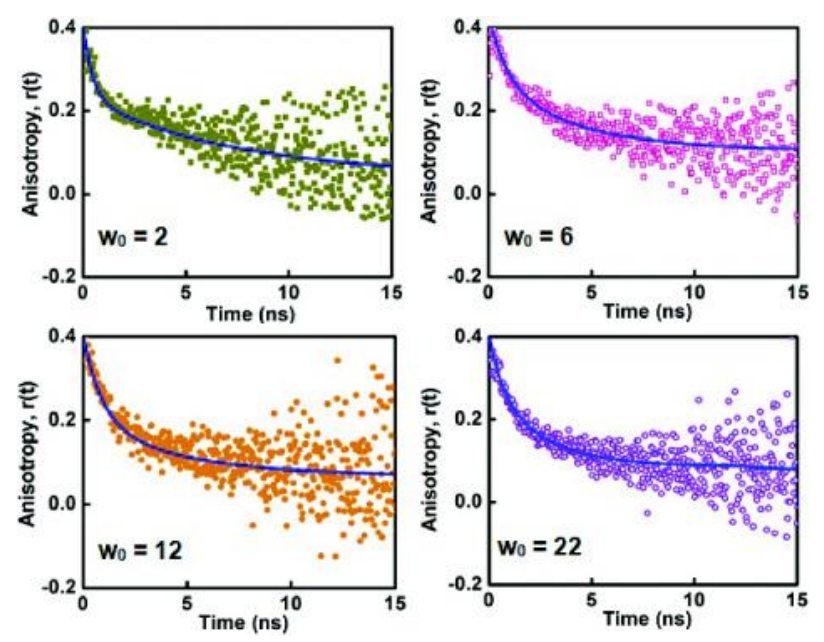

Fig. 5: Time-resolved fluorescence anisotropy decay transients of NFX $\left(\lambda_{e x}=340 \mathrm{~nm},[\mathrm{NFX}]=2.0 \mu \mathrm{M}\right)$ in AOT/n-heptane reverse micelles at various $w_{0}$ values $\left(w_{0}=2:-\square-, w_{0}=6:-\square-, w_{0}=12:-\bullet-, w_{0}=22:-0-\right)$ as specified in the figure legend

Table 2: Time-Resolved Fluorescence Anisotropy Decay Parameters of NFX in AOT Reverse Micelle with Increasing $\mathbf{w}_{\mathbf{0}}$

\begin{tabular}{lccccc}
\hline $\mathrm{w}_{0}$ & $\tau_{1 \mathrm{r}}^{\mathrm{a}}(\mathrm{ps})$ & $\tau_{2 \mathrm{r}}{ }^{\mathrm{a}}(\mathrm{ns})$ & $\alpha_{1 \mathrm{r}}$ & $\alpha_{2 \mathrm{r}}$ & $\langle\tau\rangle(\mathrm{ns})$ \\
\hline 2 & 356 & 7.25 & 0.63 & 0.37 & 2.91 \\
6 & 431 & 6.31 & 0.76 & 0.24 & 1.84 \\
12 & 527 & 3.66 & 0.84 & 0.16 & 1.03 \\
22 & 537 & 1.89 & 0.88 & 0.12 & 0.61 \\
\hline $\pm 4 \%$ & & & & &
\end{tabular}

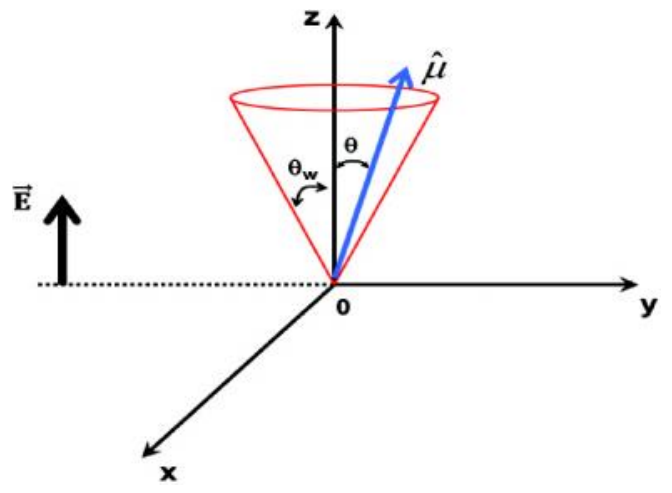

Scheme 2: Simplified ilustration of the Wobbling-in-Cone Rotation (Here, $\hat{\mu}$ signifies the symmetry axis of the flourophore, which forms an angle $\theta$ with the $z$ axis which is coincident with the orientation of the $E$ vector of the incident radiation (linearly polarized). There is unrestricted motion around the $\hat{\mu}$ axis which wobbles around the $z$-axis within a cone of semi-angle, $\theta_{w}$ )

The time-resolved anisotropic decay is found to be biexponential in nature in reverse micellar assemblies of varying water pool sizes. The overall biexponential decay comprises of an ultrafast $\left(\tau_{1 \mathrm{r}}\right)$ and a slow $\left(\tau_{2 \mathrm{r}}\right)$ component indicating the occurrence of two types of dynamical processes on various time scales. This anisotropy decay is described by the following equation (Lakowicz, 1999):

$$
r(t)=r_{0} \sum_{i} \alpha_{i r} \exp \left(-t / \tau_{i r}\right)
$$

where, $\alpha_{\text {ir }}$ is the amplitude of the $\mathrm{i}^{\text {th }}$ rotational correlation time, $\tau_{\text {ir }}$ and $r_{0}$ is the limiting anisotropy that describes the inherent depolarization of the fluorophore. The average rotational relaxation time is calculated as (Lakowicz, 1999):

$$
\left\langle\tau_{r}\right\rangle=\sum_{i} \alpha_{i r} \tau_{i r}
$$

The data compiled in Table 2 reflect a noticeable decrease of the average rotational correlation time $\left(\left\langle\tau_{r}\right\rangle\right)$ of NFX (that is, enhancement of the rotational motion) with increasing $\mathrm{w}_{0}$. This indicates a gradual relaxation of the motional restriction on the AOT-bound drug molecules with increasing $\mathrm{w}_{0}$ (Kinosita et al., 1977; Paul et al., 2011), in conformity with the steadystate results as discussed earlier (Steady State Fluorescence Study Section).

In tune with numerous literature reports, such a bi-exponential fluorescence depolarization behavior 
can be rationalized on the basis of the well-known wobbling-in-cone model (Scheme 2) (Kinisita et al., 1977). According to this model, the overall anisotropy decay may arise from three independent motions:

(i) Wobbling motion of the $\operatorname{drug}\left(r_{w}(t)\right)$ with a time constant $\tau_{w}$,

(ii) Translational motion of the drug $\left(r_{D}(t)\right)$ along the surface of the macromolecule (here reverse micelle) with a time constant $\tau_{D}$, and

(iii) Overall rotation of the macromolecule $\left(r_{P}(t)\right)$ with a time constant $\tau_{P}$ (Kinosita et al., 1977).

It is often argued that involvement of more than one type of rotational motions leads to deviation of the overall fluorescence depolarization from a monoexponential pattern. According to this model, the shorter rotational correlation time $\left(\tau_{1 r}\right)$ is interpreted according to a restricted rotor model whose rotational diffusion is restricted in a potential cone of semi angle $\theta_{w}$ about an imaginary axis. The cone itself is considered to rotate on a slower time scale.

Thus, $r(t)$ is decomposed into three independent motions as (Paul et al., 2014; Kinosita et al., 1977; Paul et al., 2011; Tan et al., 2005):

$$
r(t)=r_{w}(t) r_{D}(t) r_{p}(t)
$$

Further, $r(t)$ in terms of the generalized order parameter S, is given as (Kinosita et al., 1977; Paul et al., 2011; Tan et al., 2005):

$$
\begin{aligned}
r(t)= & r_{0}\left[S^{2}+\left(1-S^{2}\right) \exp \left[-t\left(1 / \tau_{\mathrm{w}}\right)\right]\right. \\
& \exp \left[-t\left(1 / \tau_{\mathrm{D}}+1 / \tau_{\mathrm{p}}\right)\right]
\end{aligned}
$$

Thus, the various rotational dynamical parameters are related to the faster $\left(\tau_{1 \mathrm{r}}\right)$ and slower $\left(\tau_{2 \mathrm{r}}\right)$ rotational correlation time constants as follows (Paul et al., 2014; Kinosita et al., 1977; Paul et al., 2011; Tan et al., 2005):

$$
\begin{aligned}
& S=\sqrt{a_{2 r}} \\
& \frac{1}{\tau_{w}}=\frac{1}{\tau_{1 r}}-\frac{1}{\tau_{2 r}}
\end{aligned}
$$

Here, $S$ is the generalized order parameter.

Again the order parameter $(S)$ is connected to the semi angle, $\theta_{w}$ according to (Paul et al., 2014; Kinosita et al., 1977; Paul et al., 2011; Tan et al., 2005):

$$
S=\frac{1}{2} \cos \theta_{w}\left(1+\cos \theta_{w}\right)
$$

The wobbling diffusion coefficient $\left(D_{\mathrm{w}}\right)$ of NFX producing information of the cone-restricted motion of the RM-bound drug is given as (Paul et al., 2014; Kinosita et al., 1977; Paul et al., 2011; Tan et al., 2005):

$$
D_{w}=\frac{7 \theta_{w}^{2}}{24 \tau_{w}}, \text { for } \theta_{w} \leq 30^{\circ}
$$

For $\theta_{w} \geq 30^{\circ} D_{w}$ is obtained as $\left(x=\cos \theta_{w}\right)$ :

$$
\begin{aligned}
& D_{w}=\left\{\left(1-S^{2}\right) \tau_{w}\right\}^{-1} \\
& {\left[\frac{x^{2}(1+x)^{2}}{2(x-1)}\left\{\ln \left(\frac{1+x)}{2}\right)+\left(\frac{1-x)}{2}\right)\right\}\right.} \\
& \left.+\left(\frac{1-x)}{24}\right)\left(6+8 x-x^{2}-12 x^{3}-7 x^{4}\right)\right]
\end{aligned}
$$

In the present case $\left(\theta_{w}>30^{\circ}\right.$; Table 3$)$, equation 13 is used to elucidate the wobbling diffusion coefficient $\left(D_{\mathrm{w}}\right)$ as collected in Table 3 .

The calculated rotational parameters (Table 3) show the impartation motional restriction on the drug molecules within the RM-encapsulated state as compared to bulk aqueous phase, whereas the degree of motional restriction imposed on the drug molecules gradually relaxes with increasing water pool size $\left(w_{0}\right.$ value) of the RM. This is distinctly manifested by the decreasing magnitude of the order parameter $(S)$ with $w_{0}$ (Table 3 ). The physical significance of the order

Table 3: Rotational Dynamical Parameters of NFX as Calculated on the Basis of the Wobbling-in-Cone Model

\begin{tabular}{lcccc}
\hline$w_{0}$ & $\tau_{\mathrm{w}}(p s)$ & $S$ & $\theta_{w}\left({ }^{\circ}\right)$ & $D_{w} \times 10^{-8}\left(\mathrm{~s}^{-1}\right)$ \\
\hline 2 & 374 & 0.61 & 44.68 & 3.91 \\
6 & 462 & 0.49 & 52.48 & 3.93 \\
12 & 616 & 0.40 & 58.35 & 3.37 \\
22 & 750 & 0.35 & 61.9 & 2.96 \\
\hline
\end{tabular}


parameter $\left(S=\sqrt{a_{2 r}}\right)$ can be understood in terms of the degree of spatial restriction on the wobbling-incone orientational motion of the drug. The order parameter provides a physical estimate to the orientational motion of the drug within the limit: $S=0$ for unrestricted motion to $S=1$ for complete restriction. A decreasing rigidity on the drug molecules within the RMs with increasing $w_{0}$ value is reinforced from an enhanced magnitude of the semi angle $\left(\theta_{w}\right)$ (Table 3).

\section{Modulated Photophysics of NFX within AOT Reverse Micelle: Effect of pH or Polarity?}

In the present context, a natural inquisition may prevail whether the changes in the photophysical properties of NFX with increasing water pool size within the AOT reverse micelle are originating from the modulation of the polarity in the vicinity of the microenvironment of the bound drug or change of $\mathrm{pH}$ affecting the protonation/deprotonation equilibrium of NFX.

In order to resolve the issue, we have resorted upon comparison of the photophysical properties of the drug in AOT reverse micelle in which the water pool $\left(w_{0}=15\right)$ is created with (i) normal water, (ii) acidic water of $\mathrm{pH}=4.0$ and (iii) alkaline water of $\mathrm{pH}=10.0$ (Satpati et al., 2009). A detailed pHdependent study in AOT reverse micelle is, however, not very relevant given the lacuna surrounding the effect on the structure of the reverse micelle of the additives required for changing the $\mathrm{pH}$ within the water pool. Figure 6A shows that in all the three cases under investigation, the emission spectra of NFX are strikingly similar to each other (having $\lambda_{\mathrm{em}}=440 \mathrm{~nm}$ ). This observation is strongly substantiated from timeresolved fluorescence decay results (Fig. 6B) which are found to be in close proximity with each other within experimental error limit. Thus, it appears rational to conclude that the observed changes in the photophysical properties of the drug with varying water content within the AOT reverse micelle are more likely to be related to the change in polarity rather than $\mathrm{pH}$ in the microenvironment of the bound drug (Satpati et al., 2009).

\section{Conclusions}

The present work reports a spectroscopic study of the photophysical properties of NFX within reverse micellar environments as a function the water pool size. Our results reveal that with increasing water pool size inside the micro-heterogeneous reverse micellar core the spectroscopic properties of the bound drug are remarkably modified. The marked red-shift in fluorescence wavelength and decrease of fluorescence anisotropy of NFX with increasing $\mathrm{w}_{0}$ suggest that the microenvironment in the vicinity of the drug molecules tend to become more bulk like with enhanced hydration (increase in $w_{0}$ ). These results are also found to bear reasonable parity with earlier literature and are further corroborated from time-resolved measurements. The rotational dynamical parameters of NFX within AOT RM as a function of the $w_{0}$ value have been calculated using the wobbling-in-cone model which yields meticulous information regarding the degree of motional constraint imposed on the drug molecules in terms of variation of the generalized order parameter $(\mathrm{S})$ and the cone semi angle $\left(\theta_{w}\right)$. These results demonstrate the decrease of motional restriction with increase of the water pool size. Furthermore, we have tried to show that our specific experimental results demonstrate the predominating role of change of
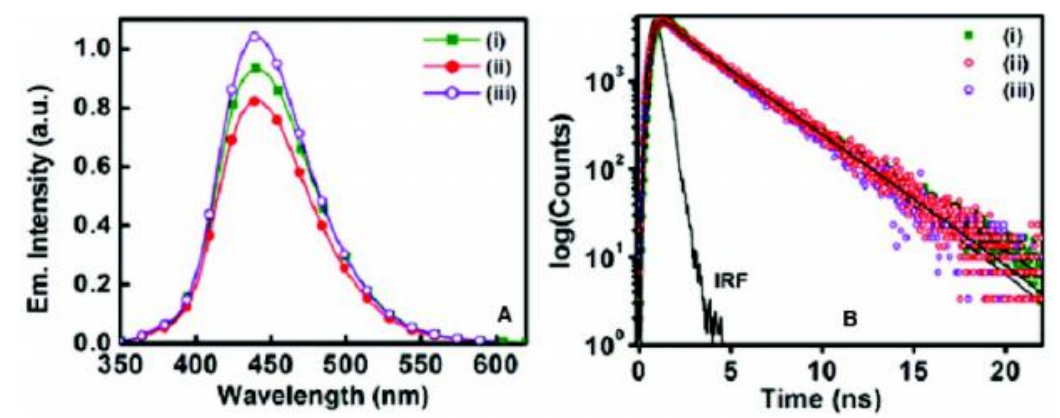

Fig. 6: Comparison of (A) fluorescence spectra and (B) time-resolved fluorescence decay transients (IRF: Instrument Response Function) of NFX in AOT/n-heptane reverse micelles at $\mathrm{w}_{0}=15$ with (i) normal water, (ii) water of $\mathrm{pH}=10.0$, and (iii) water of $\mathrm{pH}=\mathbf{4 . 0}$ 
polarity rather than $\mathrm{pH}$ in the microenvironment of the bound drug, which is responsible for the observed modulations in the photophysical properties of the drug with water pool size inside AOT reverse micelle.

\section{Graphical Abstract}

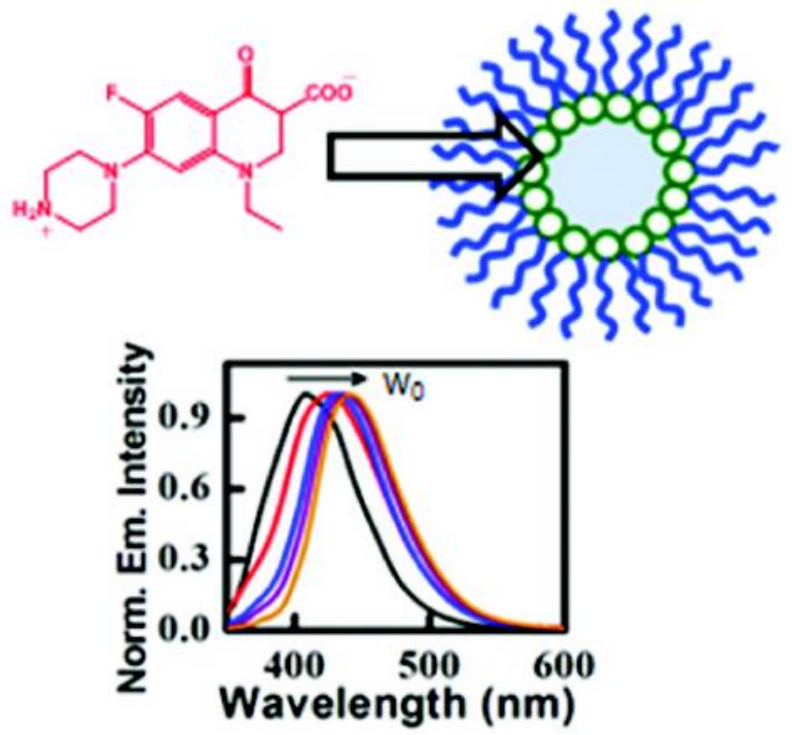

\section{Acknowledgments}

The acknowledge receipt of post-doctoral research fellowship (BKP) from IISER Bhopal, doctoral research fellowships from CSIR (NG \& AT) and funding from DST.

\section{References}

Albini A and Monti S (2003) Photophysics and photochemistry of fluoroquinolones ChemSoc Rev 32 238-250

Anand U, Kurup L and Mukherjee S (2012) Deciphering the role of $\mathrm{pH}$ in the binding of Ciprofloxacin hydrochloride to Bovine serum albumin Phys ChemChem Phys 14 42504258

Appelbaum P C and Hunter P A (2000) The fluroquinolone antibacterials: past, present and future perspectives Int $J$ Antimicrob Agents 16 5-15

Ball P (2000) Quinolone generations: natural history or natural selection? J Antimicrob Chemother 46 17-24

Baruah B, Roden J M, Sedgwick M, Correa N M, Crans D C and Levinger D E (2006) When water is not water? Exploring water confined in large reverse micelles using a highly charged inorganic molecular probe $J$ Am ChemSoc 128 12758-12765

Berlanga M, Ruiz N, Herna'ndez-Borrell, Montero T and Vinas
M (2000) Role of the outer membrane in the accumulation of quinolones by Serratiamarcescens Can J Microbiol46 716-722

Bhattacharyya K (2003) Solvation dynamics and proton transfer in supramolecular assemblies Acc Chem Resspace 36 95101

Bhattacharyya K and Bagchi B (2000) Slow dynamics of constrained water in complex geometries $J$ Phys Chem A 104 10603-10613

Biswas R, Rohman N, Pradhan T and Buchner R (2008) Intramolecular charge transfer reaction, polarity, and dielectric relaxation in AOT/water/ heptane reverse micelles: pool size dependence $J$ Phys Chem B 112 9379-9388

Blondeau J M (1999) Expanded and utility of the new fluroquinolones: a review Clin Ther 21 3-40

Dutt G B (2002) Rotational Relaxation of Hydrophobic Probes in Nonionic Reverse Micelles: Influence of Water Content on the Location and Mobility of the Probe Molecules $J$ Phys Chem B 108 805-810

Faeder J and Ladanyi B M (2008) Molecular dynamics simulations of the interior of aqueous reverse micelles $J$ Phys Chem B 104 1033-1046

Fendler J H (1976) Interactions and reactions in reversed micellar system Acc Chem Res 9 153-161

Goto A, Yoshioka H, Kishimoto H and Fujita T (1992) Calorimetric studies on the state of water in reversed micelles of sodium bis (2-ethylhexyl) sulfosuccinate in different solvents Langmuir 8 441-445

Jain T K, Varshney T K and Maitra A (1989) Structural studies of aerosol OT reverse micellar aggregates by FT-IR $J$ Phys Chem 93 7409-7416

Kalyanasundaram K (1987) Photochemistry in microheterogeneous systems. Academic Press: London

Kelkar D A and Chattopadhyay A (2004) Depth-dependent solvent relaxation in reverse micelles: a fluorescence approach $J$ Phys Chem B 108 12151-12158

Kinosita K, Kawato S and Ikegami A (1977) A theory of fluorescence polarization decay in membranes Biophys $J$ 20 289-305

Lakowicz J R (1999) Principles of fluorescence spectroscopy. Plenum: New York

Levinger N E (2002) Water in confinement Scienc e 298 17221723

Lipsky B A and Baker C A (1999) Fluoroquinolone toxicity profiles: a review focusing on newer agents Clin Infect Dis 28 352-364

Luisi P L, Giomini M, Pileni M P and Robinson B H (1988) 
Reverse micelles as host for proteins and small molecules Biochim Biophys Acta 947 209-246

Luisi P L and Straub B (1984) Reverse micelles. Plenum Press: New York

Mitra R K, Sinha S S and Pal S K (2008) Temperature dependent solvation dynamics of water in sodium bis (2-ethylhexyl) sulfosucinate/isooctane reverse micelles Langmuir $\mathbf{2 4} 49$ 56

Monti S and Sortino S (2002) Laser flash photolysis study of photoionization in fluoroquinolones Photochem Photobiol Sci 1 877-881

Nandi N, Bhattacharyya K and Bagchi B (2000) Dielectric relaxation and solvation dynamics of water in complex chemical and biological systems Chem Rev 100 2013-2046

Nave S, Eastoe J, Heenan R K, Steytlar D and Grillo I (2000) What is so special about aerosol-OT? 2. Microemulsion systems Langmuir 16 8741-8748

Nikaido H and Thanassi D G (1993) Penetration of lipophilic agents with multiple protonation sites into bacterial cells: tetracyclines and fluroquinolones as examples Antimicrob Agents Chemother 37 1393-1399

Paul B K, Ghosh N and Mukherjee S (2014) Prototropic transformation and rotational-relaxation dynamics of a biological photosensitizer norharmane inside nonionic micellar aggregates J Phys Chem B 118 11209-11219

Paul B K, Ghosh N and Mukherjee S (2015) Interplay of multiple interaction forces: binding of norfloxacin to human serum albumin J Phys Chem B 119 13093-13102

Paul B K and Guchhait N (2011) Exploring the strength, mode, dynamics, and kinetics of binding interaction of a cationic biological photosensitizer with DNA: Implication on dissociation of the drug-DNA complex via detergent sequestration J Phys Chem B 115 11938-11949
Satpati A K, Kumbhakar M, Nath S and Pal H (2009) Influence of Confined Water on the Photophysics of Dissolved Solutes in Reverse Micelles Chem Phys Chem 10 2966-2978

Sengupta A, Gavvala K, Koninti R K, Chaudhuri H and Hazra P (2013)Folding dynamics of flavin adenine dinucleotide (FAD) inside non-aqueous and aqueous reverse micelles. Chem Phys Lett 584 67-73

Sengupta B, Guharay J and Sengupta P K (2000) Characterization of the fluorescence emission properties of prodan in different reverse micellar environments Spectrochim Acta Part A 56 1433-1411

Silber J J, Biasutti A, Abuin E and Lissi E (1999) Interaction of small molecules with reverse micelle $A d v$ Colloid Interface Sci 82 189-252

Sortino S, Guidi G D, Giuffrida S, Monti S and VelarditaA(1998) pH effects on spectroscopic and photochemical behaviour of enoxacin: a steady state and time resolved study Photochem Photobiol 67 167-173

Stahlmann R and Lode H (1999) Toxicity of quinolones Drugs 58 37-42

Tan H S, Piletic I R and Fayer M D (2005) Orientational dynamics of water confined on a nanometer length scale in reverse micelles J Chem phys 122 174501-174509

Ting Z, Jung-Ling L, Xiao-Chao M, Jian X and Zeng-Hong T (2003) Reliability of phototoxic test of fluoroquinolones in vitro Acta Pharmacol Sin 24 453-459

Venables D S, Huang K and Schmuttenmaer C A (2001) Effect of reverse micelle size on the vibrational band of confined water and methanol J Phys Chem B 105 9132-9138

Willard D M, Riter R E and Levinger N E (1998) Dynamics of polar solvation in lecithin/water/cyclohexane J Am Chem Soc 120 4151-4160. 